\title{
Gemcitabine and S-1 Induction Chemotherapy Followed by Chemoradiotherapy for Locally Advanced Pancreatic Cancers
}

\author{
KOSEI MAEMURA ${ }^{1}$, YUKO MATAKI ${ }^{1}$, HIROSHI KURAHARA ${ }^{1}$, YOTA KAWASAKI ${ }^{1}$, SATOSHI IINO ${ }^{1}$, \\ MASAHIKO SAKODA ${ }^{1}$, YASUTO UCHIKADO ${ }^{1}$, TAKAAKI ARIGAMI ${ }^{1}$, YOSHIKAZU UENOSONO ${ }^{1}$, \\ SHINICHIRO MORI ${ }^{1}$, SHINICHI UENO ${ }^{2}$, HIROYUKI SHINCHI ${ }^{3}$ and SHOJI NATSUGOE ${ }^{1}$ \\ ${ }^{1}$ Department of Digestive Surgery, Breast and Thyroid Surgery, Kagoshima University, Kagoshima, Japan; \\ ${ }^{2}$ Clinical Oncology, Kagoshima University, Kagoshima, Japan; \\ ${ }^{3}$ Graduate School of Health Sciences, Kagoshima University, Kagoshima, Japan
}

\begin{abstract}
Background: Gemcitabine and S-1 are drugs commonly used for treating locally advanced pancreatic cancer (LAPC). However, the safety and efficacy of combination of these agents for induction chemotherapy (IC) followed by chemoradiotherapy are not well-defined. Patients and Methods: Fifteen patients with LAPC (IC-CRT group) were treated with gemcitabine and S-1 IC, followed by $S-1$ chemoradiotherapy. The clinical outcomes were compared to a cohort of 38 patients who received chemoradiotherapy alone (CRT group). Results: Disease control rates in the CRT and IC-CRT groups were $84 \%$ and $93 \%$, respectively $(p=0.024)$. The median time of disease progression was 10.8 and 15.4 months in the CRT and IC-CRT group, respectively ( $p=0.043)$. The median overall survival time was longer in the IC-CRT group compared to CRT (23.4 vs. 17.3 months), but this increase was not statistically significant. Conclusion: Gemcitabine and S-1 combination chemotherapy, followed by $C R T$, is a promising IC regimen for treating LAPC.
\end{abstract}

Pancreatic cancer is well known for its extremely unfavorable prognoses and it is the fourth leading cause of cancer death, with an overall 5-year survival rate of only $1-4 \%$ (1). More than $80 \%$ of patients have a locally advanced or metastatic form of the disease, that cannot be treated with surgical resection (2).

There are a number of palliative options for unresectable pancreatic cancers, such as chemotherapy, chemoradiotherapy

Correspondence to: Kosei Maemura, MD, Ph.D., Department of Digestive Surgery, Breast and Thyroid Surgery, Kagoshima University Graduate School of Medical and Dental Sciences, 8-351, Sakuragaoka, Kagoshima 890-8520, Japan. Tel: +81 992755361, Fax: +81992657426, e-mail: kmaemura@hotmail.co.jp

Key Words: Induction chemotherapy, chemoradiotherapy, pancreatic cancer, S-1, gemcitabine.
(CRT) or chemotherapy followed by CRT. CRT is now generally accepted as standard treatment for locally advanced pancreatic cancer (LAPC) $(3,4)$. A combination chemotherapy using gemcitabine and fluorouracil drugs, such as S1 , is considered to be one of the more promising treatment regimens for unresectable pancreatic cancer $(5,6)$. In contrast, the exact role of CRT in combination therapy is not well understood yet, especially when compared to monochemotherapy. Several studies have examined induction chemotherapy (IC) followed by CRT as a treatment option for pancreatic cancers and have highlighted some of its potential benefits $(7,8)$. Although several studies have addressed the feasibility issues associated with the administration of IC before CRT, including S-1 therapy, the efficacy of this approach is still somewhat controversial $(9,10)$. Moreover, only a few reports exist reporting gemcitabine and S-1 (GEM/S-1) combination chemotherapy followed by CRT, as an efficacious method for treating pancreatic cancers (11).

In this study, we aimed to examine the clinical outcomes in patients with locally advanced unresectable pancreatic cancer, who had been treated with GEM/S-1 IC, followed by S-1 CRT.

\section{Patients and Methods}

Patients. This study was conducted in accordance with the guidelines outlined in the Declaration of Helsinki. Fifty-three patients with histologically or cytologically confirmed adenocarcinomas were enrolled for this study at the Kagoshima University Hospital in Kagoshima, Japan, between January 2008 and December 2014. Eligible patients had to be older than 20 years of age, possess a Karnofsky performance score (KPS) (12) greater than 70 , and not to have received prior radiotherapy or chemotherapy for another malignancy within the past five years. All patients in the study cohort had locally advanced and unresectable pancreatic cancers. Our criteria for identifying locally advanced and unresectable tumors were as follows: tumor infiltration into the hepatic artery, superior mesenteric artery or celiac axis and/or unreconstructable superior mesenteric vein/portal vein occlusion. 
The tumors were determined to be inoperable owing to vascular encasement as determined by computed tomography (CT) and magnetic resonance imaging (MRI). All patients had acceptable baseline hematological, hepatic, and renal function.

Treatment schedule. IC was performed using a combination of GEM/S-1. Patients received at least two cycles of chemotherapy before CRT. Gemcitabine was administered once via $30 \mathrm{~min}$ intravenous infusions of $1000 \mathrm{mg} / \mathrm{m}^{2}$ (during day 1 and day 8) and S-1 (Taiho Pharmaceutical Co., Ltd. Tokyo, Japan) was administered orally twice a day at a dose of $40 \mathrm{mg} / \mathrm{m}^{2}$ (from day 1 to day 14).

CRT treatment was carried out for 4 weeks. Conformal radiotherapy, using 10 megavolt photons, was performed at 1.4 Gy/fractions twice each day, delivering a total dose of 56 Gy. S-1 was administered orally in two daily doses of $80 \mathrm{mg} / \mathrm{m}^{2} /$ day between days 1 and 21 . The radiation field included the primary tumor and a margin of $1-3 \mathrm{~cm}$ covering the regional lymph nodes. One month after the completion of CRT, S-1 was administered for 14 days, followed by a 7-day rest period. This cycle was repeated as a maintenance therapy until disease progression or unacceptable toxicity occurred.

Evaluation and statistical analysis. After IC completion and following 6-8 weeks; since the completion time of CRT, the clinical response of the patient was assessed by CT or MRI imaging. Tumor response was assessed according to the Response Evaluation Criteria in Solid Tumors (RECIST) (13). Treatment toxicities were recorded according to the National Cancer Institute Common Terminology Criteria for Adverse Events, version 4.0 (14). Following treatment completion, patients underwent a physical examination and a repeat testing for tumor marker levels (CA19-9; carbohydrate antigen 19-9 and CEA; carcinoembryonic antigen) as well as surveillance CT or MRI scanning every three months until disease progression occurred.

Overall survival, from the start of primary chemotherapy and CRT, was estimated using the Kaplan-Meier method. Time to progression (TTP) at the primary tumor site or distant sites was also estimated. All of the tests were conducted at an alpha level of 0.05 with a $95 \%$ confidence interval (CI). All of the statistical analyses were performed using the SPSS Statistics v17.0 (SAS Institute, Tokyo, Japan).

\section{Results}

Patient characteristics. The initial 38 consecutive patients were enrolled in the CRT group and the next 15 consecutive patients were enrolled in the IC followed by CRT group (ICCRT). Patient characteristics are summarized in Table I. Age, sex ratio, KPS, tumor size, and preoperative tumor marker levels were not found to be statistically significant different between the groups.

Result of induction chemotherapy. The average number of cycles for GEM/S-1 combination chemotherapy was 2.1 (range, 2-5 cycles). Two patients halted gemcitabine treatment owing to a hematological adverse effect in the first cycle. Administration of S-1 was performed for another three cycles to these patients. Although eight patients displayed no
Table I. Characteristics of the enrolled patients.

\begin{tabular}{lccc}
\hline & CRT $(\mathrm{N}=38)$ & IC-CRT $(\mathrm{N}=15)$ & $p$-Value \\
\hline Age (years) & & & N.S. \\
$\quad$ Range & $37-82$ & $43-83$ & \\
$\quad \begin{array}{l}\text { Median } \\
\text { Gender }\end{array}$ & 63 & 66 & \\
$\quad$ Male & 24 & & N.S. \\
Female & 14 & 8 & \\
KPS (mean \pm SD) & $86 \pm 5.2$ & $85 \pm 6.3$ & N.S. \\
Tumor site & & & N.S. \\
Head & 29 & 13 & \\
Body and tail & 9 & 2 & \\
Tumor size (mean $\pm S D)$ & $3.5 \pm 0.69$ & $3.3 \pm 0.83$ & N.S. \\
Tumor marker & & & \\
CEA (mean \pm SD) & $5 \pm 3.6$ & $4.8 \pm 4.9$ & N.S. \\
CA19-9 (mean $\pm S D)$ & $769 \pm 1389$ & $215 \pm 291$ & N.S. \\
\hline
\end{tabular}

KPS, Karnofsky performance status; N.S., not significant.

toxicities, grade 3 neutropenia occurred in three patients, and anemia occurred in one patient. Grade 4 of toxicity was not observed in any patient (Table II).

As two patients achieved a partial response, nine patients displayed stable disease and two patients displayed progressive disease, disease control rate after IC was $86 \%$ in this group. Distant metastasis did not appear in any patients following IC. All patients were consecutively treated with CRT.

Results of chemoradiotherapy. All patients, in both groups, received a scheduled dose of radiotherapy. Toxicities during and after CRT treatment, in both groups are shown in Table II. Although the incidence of hematological toxicities was higher in the IC-CRT group, no grade 4 toxicities were observed in either group. Three patients displayed grade 3 neutropenia, two in the CRT group and one in the IC-CRT group. Febrile neutropenia was not seen in our study cohort. The non-hematological toxicities observed were relatively mild and no grade 4 toxicities were recorded.

Tumor response and tumor reduction rate based on radiological assessment and CEA/CA19-9 expression levels are shown in Table III. Disease control rate was 84\% (32 patients out of 38) in the CRT group and 93\% (14 patients out of 15) in the IC-CRT group. The average reduction rate in tumor size was $17.7 \%$ in the CRT group and $29.9 \%$ in the IC-CRT group after the treatment. Metastatic lesions appeared in four patients, one of whom belonged to the ICCRT group. Decreased carcinoembryonic antigen (CEA) levels were observed in 35 patients, 25 of the CRT group and 10 of the IC-CRT group. Decreased CA19-9 levels were seen in 41 patients, 28 of the CRT group and 13 of the IC-CRT group. The difference between the radiological responses in 
Table II. Toxicities during induction chemotherapy and chemoradiotherapy.

\begin{tabular}{|c|c|c|c|c|c|c|c|c|c|}
\hline \multirow{3}{*}{ Toxicity } & \multicolumn{4}{|c|}{ CRT } & \multicolumn{5}{|c|}{ IC-CRT } \\
\hline & \multicolumn{3}{|c|}{ Grade } & \multicolumn{3}{|c|}{$\begin{array}{c}\text { IC } \\
\text { Grade }\end{array}$} & \multicolumn{3}{|c|}{$\begin{array}{l}\text { CRT } \\
\text { Grade }\end{array}$} \\
\hline & 2 & 3 & 4 & 2 & 3 & 4 & 2 & 3 & 4 \\
\hline \multicolumn{10}{|l|}{ Hematological } \\
\hline Leukopenia & 3 & 2 & 0 & 0 & 0 & 0 & 2 & 3 & 0 \\
\hline Neutropenia & 2 & 2 & 0 & 0 & 3 & 0 & 2 & 1 & 0 \\
\hline Anemia & 0 & 0 & 0 & 0 & 1 & 0 & 0 & 1 & 0 \\
\hline Thrombocytopenia & 0 & 0 & 0 & 2 & 0 & 0 & 1 & 1 & 0 \\
\hline \multicolumn{10}{|l|}{ Non-hematological } \\
\hline Nausea & 0 & 0 & 0 & 1 & 0 & 0 & 1 & 0 & 0 \\
\hline Anorexia & 2 & 0 & 0 & 2 & 0 & 0 & 3 & 0 & 0 \\
\hline Vomiting & 1 & 0 & 0 & 0 & 0 & 0 & 0 & 0 & 0 \\
\hline Diarrhea & 1 & 0 & 0 & 0 & 0 & 0 & 0 & 0 & 0 \\
\hline Oral mucositis & 1 & 0 & 0 & 0 & 0 & 0 & 0 & 0 & 0 \\
\hline
\end{tabular}

Table III. Response data of radiological findings and tumor markers.

\begin{tabular}{lccc}
\hline Objective response & CRT & IC-CRT & \\
\hline Radiological response & & & $p<0.05$ \\
$\quad$ Partial response & 6 & 8 & \\
$\quad$ Stable disease & 26 & 6 & \\
$\quad$ Progressive disease & 6 & 1 & N.S. \\
Tumor reduction rate (\%) & & & N.S. \\
$\quad$ Mean (range) & $17.7(-25 \sim 70)$ & $29.9(-5 \sim 63)$ & \\
CEA response & & & \\
$\quad \geq 50 \%$ decrease & 19 & 2 & N.S. \\
$\quad<50 \%$ decrease & 6 & 5 & \\
Increase & 9 & & \\
CA19-9 response & & 4 & \\
$\quad \geq 50 \%$ decrease & 7 & 2 & \\
$\quad<50 \%$ decrease & 21 & & \\
Increase & 5 & & \\
\hline
\end{tabular}

N.S., Not significant.

the two groups was statistically significant $(p=0.024)$. However, the differences between the CEA levels and CA199 levels in the two groups were not statistically significant.

Treatment outcomes. The median TTP was 10.8 months in the CRT group and 15.4 months in the IC-CRT group (Figure 1A). Eight patients showed no disease progression. A statistically significant difference in TTP was seen between the two groups $(p=0.043)$. The median OS was 17.3 months in the CRT group and 23.4 months in the IC-CRT group (Figure 1B). Estimated 1-, 2-, and 5-year survival rates in the

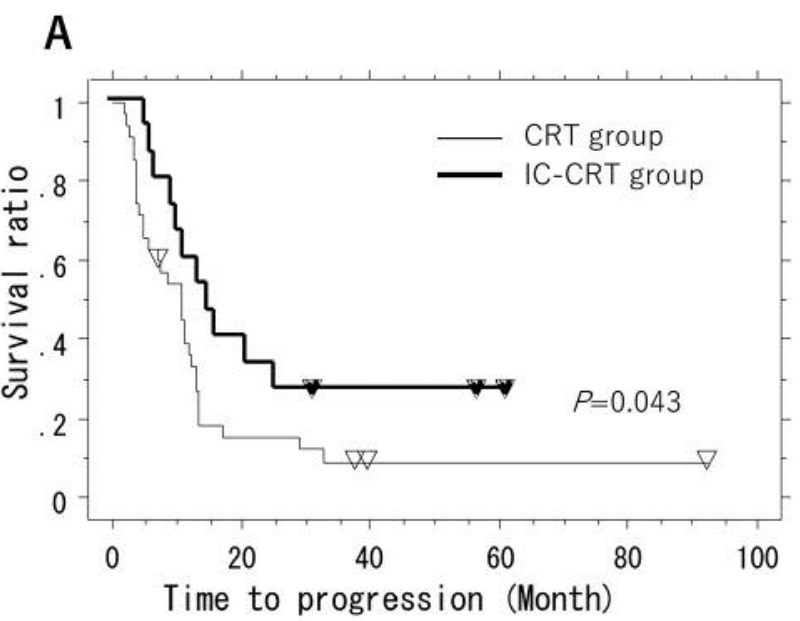

\section{B}

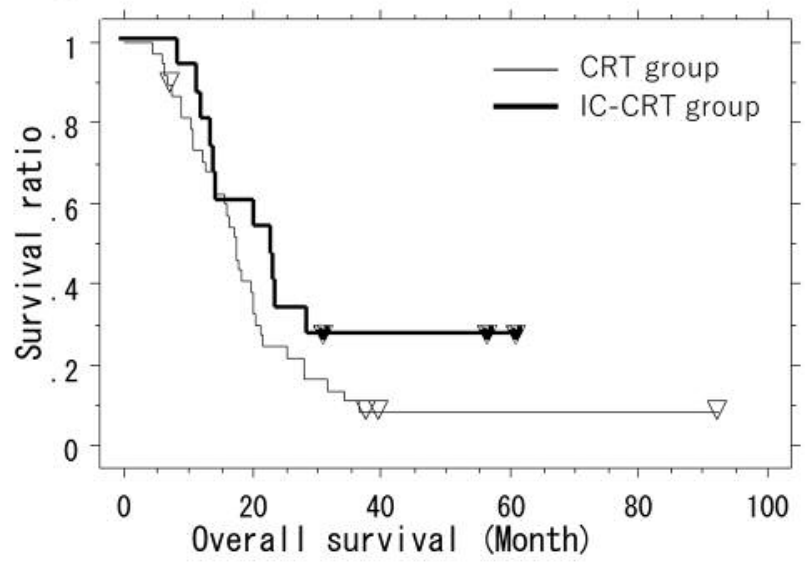

Figure 1. Kaplan-Meier plots of (A) time to progression and (B) overall survival between the CRT group and IC-CRT group. p-Value of $>0.05$ indicates statistically significant difference between groups.

CRT group were $70.5 \%, 21.7 \%$, and $8 \%$, respectively. Corresponding survival rates in the IC-CRT group were $80 \%, 33.3 \%$, and $30 \%$, respectively. At the time of analysis, 36 patients of the CRT group and 11 patients of the IC-CRT group had died. However, no statistically significant difference in the OS was observed between the two groups.

\section{Discussion}

The optimal treatment for patients with LAPC is not well established yet. The American Society of Clinical Oncology recommends that systemic therapy with combination regimens should be used as the initial method of treatment for most patients with $\operatorname{LAPC}(15,21)$. Although there are many reports that assess the efficacy of CRT in patients with 
LAPC, the exact role of CRT in combination therapies remains controversial. Recent retrospective and phase II studies have demonstrated the viability of pre-CRT IC for borderline resectable tumors and patients with LAPC $(8,16$, $17,18)$. These studies have also shown that the median OS in patients with LAPC ranges between 9.3 and 19.2 months and conversion rate for resection was in $30-40 \%$ of the patients. Therefore, evidence seems to indicate that initial systemic therapy could potentially avoid the rapid systemic dissemination of cancer. On the other hand, the LAP07 trial, which is the first phase III study on the subject, showed that CRT did not result in increased survival rates when compared to mono-chemotherapy (19). The reason behind this observed lack of survival benefit in the LAP07 trial may be the insufficient efficacy of IC.

Both, phase III trials and MPACT trial have demonstrated that FOLFIRINOX and nab-paclitaxel can significantly improve survival in patients with stage IV pancreatic cancer $(20,21)$. Sadot et al. reported that patients with LAPC treated using FOLFIRINOX had a median progression-free survival of 16 months and a median OS of 25 months (22). Although the median OS was not found to differ significantly in the LAP07 trial, CRT shown to be more effective at decreasing local progression than mono-chemotherapy. A recent systemic review showed that FOLFIRINOX-treated patients with LAPC had a median OS of 24.2 months (23). The median OS in this study, was 23 months and it is similar to that reported in the aforementioned study. These results indicate that powerful IC may improve the ability of patients to proceed to a definitive treatment that allows for local control of the disease. Therefore, radiotherapy may play an important role in increasing survival via local disease control, if distant disease control can be improved through superior systemic regimens.

Several meta-analysis studies indicate that GEM/S-1 combination therapy, as a first-line treatment, may be more effective at increasing survival rates in patients with unresectable pancreatic cancer $(5,24)$. Qing-Hua et al. showed that GEM/S-1 combination therapy followed by oral S-1 with intensity-modulated radiotherapy (IMRT) protocol achieved a 2-year survival rate of $34.4 \%$, which was similar to the results we have outlined in this study (2-year survival rate of $33.3 \%$ ) (11). In addition, our data showed that GEM/S-1 combination therapy achieved a disease control rate of $86 \%$. However, further consideration of the duration of IC is required to enhance the efficacy of this treatment strategy.

IC using S-1 based regimens was convenient for our protocol as we also conducted CRT using hyper-fractionated radiotherapy (HART) with $\mathrm{S}-1$ in this series. Our previous studies showed that HART with S-1 was well-tolerated by patients with LAPC and resulted in promising outcomes (25). Moreover, most patients received S-1 mono-therapy as a maintenance therapy. The JASPAC 01 study showed that adjuvant chemotherapy with S-1 results in significant survival benefits in patients with resected pancreatic cancer when compared with gemcitabine therapy (26). We speculate that such a regimen could result in a longer patient survival and fewer post-CRT adverse effects.

However, this study has several limitations. As this analysis was not a randomized control study, all patients were enrolled in this protocol consecutively and without allocation. Furthermore, owing to the small sample size, we cannot clearly ensure the viability of this treatment strategy. Another limitation was that the administrated dose intensity of GEM/S-1 differed amongst patients of the IC-CRT group. Considering this, a randomized control study is necessary to validate our findings.

In summary, our data suggests that GEM/S-1 IC, followed by $\mathrm{S}-1 \mathrm{CRT}$, is capable of significantly suppressing disease progression, while presenting a manageable toxicity profile. However, a multicenter randomized control trial should be conducted to confirm its survival benefits and select the proper populations.

\section{References}

1 Torre LA, Bray F, Siegel RL, Ferlay J, Lortet-Tieulent J and Jemal A: Global cancer statistics. CA Cancer J Clin 65: 87-108, 2015.

2 Egawa S, Toma H, Ohigashi H, Okusaka T, Nakao A, Hatori T, Maguchi H, Yanagisawa A and Tanaka M: Japan Pancreatic Cancer Registry; 30th year anniversary. Japan Pancreas Society. Pancreas 41: 985-992, 2012.

3 Sultana A, Tudur Smith C, Cunningham D, Starling N, Tait D, Neoptolemos JP, and Ghaneh P: Systematic review, including meta-analyses, on the management of locally advanced pancreatic cancer using radiation/combined modality therapy. $\mathrm{Br}$ J Cancer 96: 1183-1190, 2007.

4 Shinchi H, Maemura K, Mataki Y, Kurahara H, Sakoda M, Ueno S, Hiraki Y, Nakajo M, Natsugoe S and Takao S: A phase II study of oral S-1 with concurrent radiotherapy followed by chemotherapy with S-1 alone for locally advanced pancreatic cancer. J Hepatobiliary Pancreat Sci 19: 152-158, 2012.

5 Cao $\mathrm{C}$, Kuang $\mathrm{M}, \mathrm{Xu}$ W, Zhang $\mathrm{X}$, Chen $\mathrm{J}$ and Tang $\mathrm{C}$ : Gemcitabine plus S-1: a hopeful frontline treatment for Asian patients with unresectable advanced pancreatic cancer. Jpn J Clin Oncol 45: 1122-1130, 2015.

6 Li Q, Yan H, Liu W, Zhen H, Yang Y and Cao B: Efficacy and safety of gemcitabine-fluorouracil combination therapy in the management of advanced pancreatic cancer: a meta-analysis of randomized controlled trials. PLoS One 9: e104346, 2014. Available from: http://www.ncbi.nlm.nih.gov/pubmed/25093849

7 Badiyan SN, Olsen JR, Lee AY, Yano M, Menias CO, Khwaja S, Wang-Gillam A, Strasberg SM, Hawkins WG, Linehan DC, Myerson RJ and Parikh PJ: Induction chemotherapy followed by concurrent full-dose gemcitabine and intensity-modulated radiation therapy for borderline resectable and locally advanced pancreatic adenocarcinoma. Am J Clin Oncol 39: 1-7, 2016.

8 Esnaola NF, Chaudhary UB, O'Brien P, Garrett-Mayer E, Camp ER, Thomas MB, Cole DJ, Montero AJ, Hoffman BJ, Romagnuolo J, Orwat KP and Marshall DT: Phase 2 trial of 
induction gemcitabine, oxaliplatin, and cetuximab followed by selective capecitabine-based chemoradiation in patients with borderline resectable or unresectable locally advanced pancreatic cancer. Int J Radiat Oncol Biol Phys 88: 837-844, 2014.

9 Kim HR, Lee CG, Choi EC, Kim JH, Koh YW and Cho BC: Induction docetaxel and S-1 followed by concomitant radiotherapy with low-dose daily cisplatin in locally advanced head and neck carcinoma. Head Neck 38: E1653-1659, 2016.

10 Yoon DH, Jang G, Kim JH, Kim Y-H, Kim JY, Kim HR, Jung HY, Lee GH, Song HY, Cho KJ, Ryu JS and Kim SB: Randomized phase 2 trial of $\mathrm{S} 1$ and oxaliplatin-based chemoradiotherapy with or without induction chemotherapy for esophageal cancer. Int J Radiat Oncol Biol Phys 91: 489-496, 2015.

11 Ke Q-H, Zhou S-Q, Yang J-Y, Du W, Liang G, Lei Y and Luo F: S-1 plus gemcitabine chemotherapy followed by concurrent radiotherapy and maintenance therapy with $S-1$ for unresectable pancreatic cancer. World J Gastroenterol 20: 13987-13992, 2014.

12. Yates JW, Chalmer B and McKegney FP: Evaluation of patients with advanced cancer using the Karnofsky performance status. Cancer 45: 2220-2224, 1980.

13 Eisenhauer EA, Therasse P, Bogaerts J, Schwartz LH, Sargent D, Ford R, Dancey J, Arbuck S, Gwyther S, Mooney M, Rubinstein L, Shankar L, Dodd L, Kaplan R, Lacombe D and Verweij J: New response evaluation criteria in solid tumours: revised RECIST guideline (version 1.1). Eur J Cancer 45: 228247, 2009

14. National Cancer Institute: Common terminology criteria for adverse events (CTCAE) Version 4.0. Available at https://evs.nci.nih.gov/ftp1/CTCAE/CTCAE_4.03_2010-0614_QuickReference_5x7.pdf. Published May 28, 2009.

15 Balaban EP, Mangu PB, Khorana AA, Shah MA, Mukherjee S, Crane CH, Javle MM, Eads JR, Allen P, Ko AH, Engebretson A, Herman JM, Strickler JH, Benson AB 3rd, Urba S and Yee NS: Locally advanced, unresectable pancreatic cancer: American Society of Clinical Oncology Clinical Practice Guideline. J Clin Oncol 34: 2654-2668, 2016.

16 Nanda RH, El-Rayes B, Maithel SK and Landry J: Neoadjuvant modified FOLFIRINOX and chemoradiation therapy for locally advanced pancreatic cancer improves resectability. J Surg Oncol 111: 1028-1034, 2015.

17 Crane CH, Varadhachary GR, Yordy JS, Staerkel GA, Javle MM, Safran H, Haque W, Hobbs BD, Krishnan S, Fleming JB, Das P, Lee JE, Abbruzzese JL and Wolff RA: Phase II trial of cetuximab, gemcitabine, and oxaliplatin followed by chemoradiation with cetuximab for locally advanced (T4) pancreatic adenocarcinoma: correlation of Smad4(Dpc4) immunostaining with pattern of disease progression. J Clin Oncol 29: 3037-3043, 2011.

18 Huang WK, Kuo YC, Tsang NM, Hsu HC, Shen WC, Chou WC, Yang TS and Chen JS: Concurrent chemoradiotherapy with or without induction chemotherapy versus chemotherapy alone in patients with locally advanced pancreatic cancer. Anticancer Res 34: 6755-6761, 2014.
19 Hammel P, Huguet F, van Laethem J-L, Goldstein D, Glimelius B, Artru P, Borbath I, Bouché O, Shannon J, André T, Mineur L, Chibaudel B, Bonnetain F and Louvet C: Effect of chemoradiotherapy vs chemotherapy on survival in patients with locally advanced pancreatic cancer controlled after 4 months of gemcitabine with or without erlotinib: The LAP07 randomized clinical trial. JAMA 315: 1844-1853, 2016.

20 Conroy T, Desseigne F, Ychou M, Bouché O, Guimbaud R, Bécouarn Y, Adenis A, Raoul JL, Gourgou-Bourgade S, de la Fouchardière C, Bennouna J, Bachet JB, Khemissa-Akouz F, PéréVergé D, Delbaldo C, Assenat E, Chauffert B, Michel P, MontotoGrillot $\mathrm{C}$ and Ducreux M: FOLFIRINOX versus gemcitabine for metastatic pancreatic cancer. N Engl J Med 364: 1817-1825, 2011.

21 Von Hoff DD, Ervin T, Arena FP, Chiorean EG, Infante J, Moore M, Seay T, Tjulandin SA, Ma WW, Saleh MN, Harris M, Reni M, Dowden S, Laheru D, Bahary N, Ramanathan RK, Tabernero J, Hidalgo M, Goldstein D, Van Cutsem E, Wei X, Iglesias J and Renschler MF: Increased survival in pancreatic cancer with nabpaclitaxel plus gemcitabine. N Engl J Med 369: 1691-1703, 2013.

22 Sadot E, Doussot A, O'Reilly EM, Lowery MA, Goodman KA, Do RK, Tang LH, Gönen M, D'Angelica MI, DeMatteo RP, Kingham TP, Jarnagin WR and Allen PJ: FOLFIRINOX induction therapy for stage 3 pancreatic adenocarcinoma. Ann Surg Oncol 22: 3512-3521, 2015.

23 Suker M, Beumer BR, Sadot E, Marthey L, Faris JE, Mellon EA, El-Rayes BF, Wang-Gillam A, Lacy J, Hosein PJ, Moorcraft SY, Conroy T, Hohla F, Allen P, Taieb J, Hong TS, Shridhar R, Chau I, van Eijck CH and Groot Koerkamp B: FOLFIRINOX for locally advanced pancreatic cancer: a systematic review and patient-level meta-analysis. Lancet Oncol 17: 801-810, 2016.

24 Li D, Chen C, Zhou Y, Chen R, Fan X, Bi Z, Li Z and Liu Y: Gemcitabine compared with gemcitabine and s- 1 combination therapy in advanced pancreatic cancer: a systematic review and meta-analysis. Medicine (Baltimore) 94: e1345, 2015.

25 Maemura K, Shinchi H, Noma H, Mataki Y, Kurahara H, Maeda S, Hiraki Y, Nakajo M, Natsugoe S and Takao S: Comparison of hyper-fractionated accelerated and standard fractionated radiotherapy with concomitant low-dose gemcitabine for unresectable pancreatic cancer. Anticancer Res 28: 2369-2372, 2008.

26 Uesaka K, Boku N, Fukutomi A, Okamura Y, Konishi M, Matsumoto I, Kaneoka Y, Shimizu Y, Nakamori S, Sakamoto H, Morinaga S, Kainuma O, Imai K, Sata N, Hishinuma S, Ojima H, Yamaguchi R, Hirano S, Sudo T and Ohashi Y: Adjuvant chemotherapy of S-1 versus gemcitabine for resected pancreatic cancer: a phase 3, open-label, randomised, non-inferiority trial (JASPAC 01). Lancet 388: 248-257, 2016.

Received October 18, 2016

Revised November 23, 2016

Accepted November 24, 2016 